\title{
Does anxiety causes infertility or Does infertility causes
}

\section{anxiety? - A Literature review}

\section{Author: Dra. Beatriz Chambel ${ }^{1,2}$}

\section{USF Novo Mirante - ACES Loures-Odivelas; 2. Instituto de Histologia e Biologia do Desenvolvimento - Faculdade de Medicina da Universidade de Lisboa}

Background: Infertility is a very common problem. It is estimated that 1 in 8 couples have trouble getting pregnant or sustaining a pregnancy ${ }^{1}$. Anxiety and stress have been hypothesized as causing infertility for years, but the relationship never has been proved ${ }^{1}$. The evidence is conflicting but is possible that body-stress response and hormones involvement had a negative impact on fertility ${ }^{10}$.

On the other hand, the risk of depression, anxiety, and distress is well documented for infertile couples. Various studies report anxiety and depression prevalence previous to infertility treatments around $30-40 \%$. Depression, anxiety, isolation, and loss of control are frequent symptoms on these couples, and the drugs used in fertility treatments are associated with psychological symptoms such as anxiety, depression, and irritability ${ }^{1}$.

\section{Methods: \\ Research \\ on} databases (Pubmed, Cochrane library and SUMsearch) reviews articles and original articles using the MeSH terms: anxiety, infertility, causality. We include articles published in the past five years, in English or Portuguese language.

\section{Results:}

1) Distress is associated with long menstrual cycles, oocytes retrieved and decreased fertilization resulting in low pregnancy rates ${ }^{10}$.

2) Depression and anxiety symptoms affects cortisol release causing delay follicular maturation and anovulatory cycles ${ }^{10}$.

3) Women with high levels of $\alpha$-amylase levels (a stress's biomarker) during the fertile window has lower chances to conceive and higher times to pregnancy ${ }^{1,10}$.

4) Some studies correlate prolactin levels and thyroid dysfunction as probable causes, but the real causes remain unclear ${ }^{5}$.
1) Dealing with infertility is associated with periodically higher levels of distress and anxiety ${ }^{8}$. Women often report feelings of loss, grief, anger, and sadness. Experience a lack of femininity, shame, self-blame, and other negative emotions is common ${ }^{9}$.

2) Infertility had a serious impact on the couple's sexuality and marital harmony ${ }^{3}$. In infertile women, older ages are associated with the highest rates of depression 4

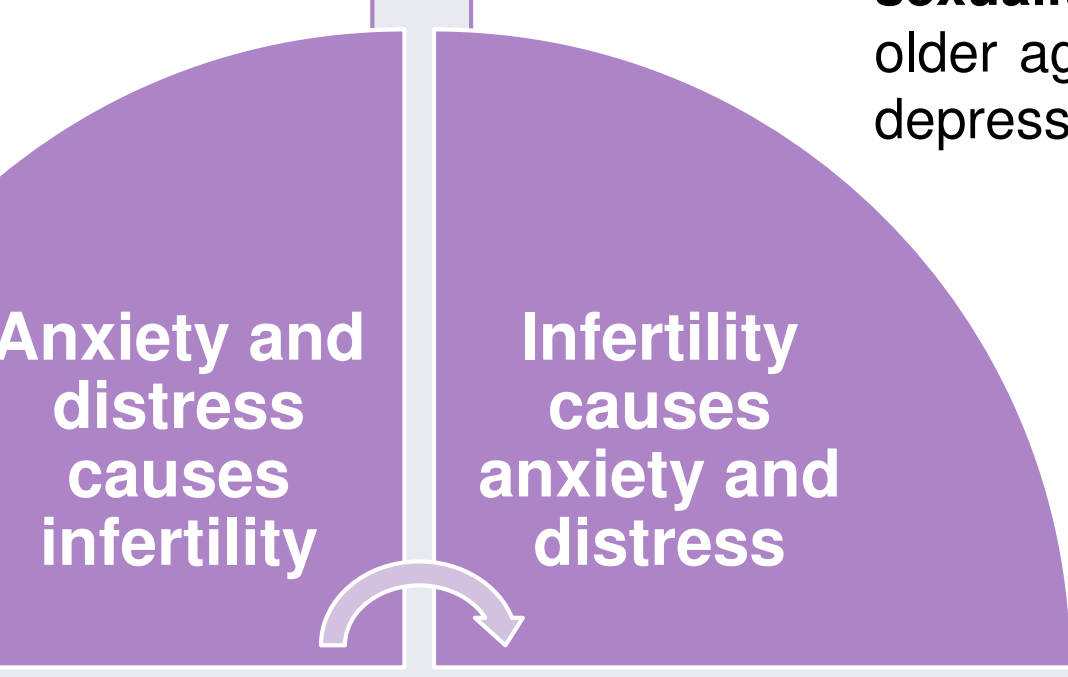

3) Psychological distress and anxiety are more common among women than among men $^{6}$, but men are also affected. Many of then experience sexual dysfunction in relation to the inability to conceive ${ }^{2}$.
1) It is clear that psychological interventions to reduce emotional distress has a positive impact on pregnancy rates $^{1}$. Pharmacological interventions do not increase pregnancy rates ${ }^{9}$.

2) Negative feelings such as anger, grief, sadness, and self-blame are the major reason to stop ART ${ }^{9}$.

3) A meta-analysis with 39 studies regarding psychological interventions shows that cognitive-behavioral therapy and mind/body interventions are beneficial for reducing distress and improving pregnancy outcomes of $\mathrm{ART}^{8}$. Music therapy combining with herbal medicine, psychotherapy, massage, and aromatherapy increases the probability of success ${ }^{7}$
Psychological

interventions

III

Conclusions:

$\checkmark$ There are not enough studies to establish a causal directionality between anxiety and infertility.

$\checkmark$ Body stress response and hormone involvement appear to be connected with infertility pathophysiology, but the mechanism itself remains unclear.

$\checkmark$ Patients who experience infertility report more anxiety and depression symptoms and that appear to be connected with ART outcomes.

$\checkmark$ Psychological interventions are beneficial for reducing distress symptoms and improve pregnancy rates and should be offered to every couple. 